\title{
Pool Boiling Enhancement with SLM Producing Grid Structures
}

\author{
Chi Zhang1, Li Zhang1, Hong Xu', Pei Li², Bo Qian² \\ ${ }^{1}$ State-Key Laboratory of Chemical Engineering, School of Mechanical and Power Engineering, East China University of \\ Science and Technology \\ No. 130 Meilong Road Xuhui District, Shanghai, China \\ czhang@mail.ecust.edu.cn; lzhang@ecust.edu.cn \\ ${ }^{1}$ School of Mechanical and Power Engineering, East China University of Science and Technology \\ No. 130 Meilong Road Xuhui District, Shanghai, China
}

\section{Extended Abstract}

In recent years, more and more techniques and theories have been proposed to enhance and predict pool boiling heat transfer. Various surface modifications proved their ability in increasing boiling heat transfer through extended surface area, increased nucleation site density, wettability, capillary wicking, wavelength variation and so on. Uniform porous coatings have been proposed by Chang and You [1] since 1997 and then have been investigated by many other researchers due to its remarkable pool boiling enhancement. Recently non-uniform porous or structured surfaces have been reported to further increase the enhancement limit of uniform porous structures. Liter and Kaviany [2] prepared modulated porous surfaces using graphite mold and increased CHF to 3 times of the plain surface. Jaikumar and Kandlikar [3] combined surface microchannel with sintered microporous coatings. A maximum CHF of $420 \mathrm{~W} / \mathrm{cm} 2$ was achieved. Bai et al. [4] proposed the phase separation and modulation concept and designed a new porous artery structure with square channels. A CHF of $610 \mathrm{~W} / \mathrm{cm} 2$ was achieved.

In this paper, grid structures were manufactured with the selective laser melting (SLM) technique for boiling heat transfer enhancement. The SLM technique has specially advantages in producing complex 3D structures and parameters. The grid structures were designed with specific parameters such as grid width and thickness. The line scan spacing method was adopted to produce various grid width and thickness structures. The most commercially used 316L stainless steel powder was chosen as the building material. Saturated pool boiling experiment was then conducted on a ceramic heater to manifest its performance in enhancing boiling heat transfer. The grid structure was tightened on a ceramic heater with two stainless steel threads.

The results show grid structures could effectively enhance nucleate boiling heat transfer coefficient and critical heat flux (CHF). The heat transfer coefficient first increased when grid width was increased from 0.4 to $0.7 \mathrm{~mm}$ and then decreased with increasing grid width. For small grid channels, increased grid width decreased liquid vapour flow resistance and thus increased heat transfer coefficient. When the channels are large enough, they are flooded with water and can no longer serve as nucleation sites. Whereas, the CHF increased monotonically with grid width. Around three times CHF enhancement was observed for 1.1 and $1.3 \mathrm{~mm}$ grid width structures. The results are almost the same as the results obtained by Suazlan and Mori [5] that combined two enhancement method. The enhancement was attributed to the grid structure's "partition effect" that inhibit Helmholtz instability, confine bubble growth and hot spot expansion in the near surface region at high heat fluxes. The results given important information on future 3D porous structure design: should combine dimensions of different scale like fractals. Such structure combines the advantage of different scales that could maximize the potential of a single surface modification technique through modulation, phase separation and minimize liquid vapor flow resistance. And with SLM technique any complex 3D structure is easy to build.

\section{References}

[1] J. Y. Chang and S. M. You, "Boiling heat transfer phenomena from microporous and porous surfaces in saturated FC72," International Journal of Heat and Mass Transfer, vol. 40, no. 18, pp. 4437-4447, 1997.

[2] S. G. Liter and M. Kaviany, "Pool-boiling CHF enhancement by modulated porous-layer coating: theory and experiment," International Journal of Heat and Mass Transfer, vol. 44, no. 22, pp. 4287-4311, 2001. 
[3] A. Jaikumar and S. G. Kandlikar, "Ultra-high pool boiling performance and effect of channel width with selectively coated open microchannels," International Journal of Heat and Mass Transfer, vol. 95, pp. 795-805, 2016.

[4] L. Bai, L. Zhang, G. Lin, and G. P. Peterson, "Pool boiling with high heat flux enabled by a porous artery structure," Applied Physics Letters, vol. 108, no. 23, p. 233901, 2016.

[5] S. Mt Aznam, S. Mori, F. Sakakibara, and K. Okuyama, "Effects of heater orientation on critical heat flux for nanoparticle-deposited surface with honeycomb porous plate attachment in saturated pool boiling of water," International Journal of Heat and Mass Transfer, vol. 102, pp. 1345-1355, 2016. 